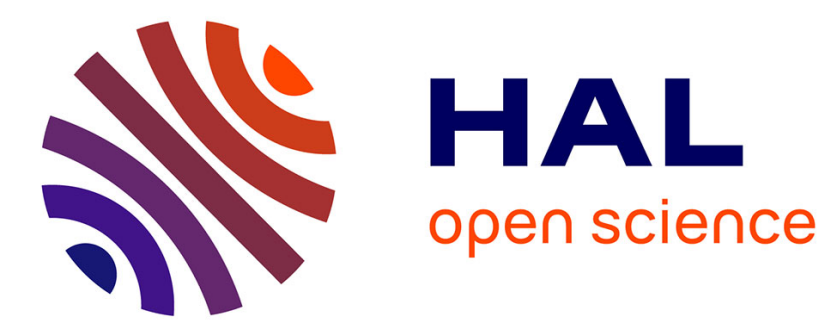

\title{
Étude par R. P. E. de la variation thermique du couplage hyperfin de Mn2+ dans CdF2
}

\author{
G. Bacquet, M. Bonnet, J. Dugas, C. Escribe
}

\section{To cite this version:}

G. Bacquet, M. Bonnet, J. Dugas, C. Escribe. Étude par R. P. E. de la variation thermique du couplage hyperfin de Mn2+ dans CdF2. Revue de Physique Appliquée, 1971, 6 (2), pp.181-182. 10.1051/rphysap:0197100602018100 . jpa-00243519

\section{HAL Id: jpa-00243519 https://hal.science/jpa-00243519}

Submitted on 1 Jan 1971

HAL is a multi-disciplinary open access archive for the deposit and dissemination of scientific research documents, whether they are published or not. The documents may come from teaching and research institutions in France or abroad, or from public or private research centers.
L'archive ouverte pluridisciplinaire HAL, est destinée au dépôt et à la diffusion de documents scientifiques de niveau recherche, publiés ou non, émanant des établissements d'enseignement et de recherche français ou étrangers, des laboratoires publics ou privés. 


\title{
ÉTUDE PAR R. P. E. DE LA VARIATION THERMIQUE DU COUPLAGE HYPERFIN DE $\mathrm{Mn}^{2+}$ DANS $\mathrm{CdF}_{2}$
}

\author{
G. BACQUET, M. BONNET, J. DUGAS et C. ESCRIBE \\ Laboratoire de Physique des Solides, Associé au C. N. R. S. \\ 118 , route de Narbonne, 31 , Toulouse
}

\begin{abstract}
Résumé. - Nous avons étudié par R. P. E. la variation, en fonction de la température, de la constante de couplage hyperfin de $\mathrm{Mn}^{2+}$ dans $\mathrm{CdF}_{2}$. Cette variation a été interprétée à l'aide de la théorie de Šimanek et Orbach.
\end{abstract}

\begin{abstract}
The temperature dependence of hyperfine coupling constant of $\mathrm{Mn}^{2+}$ in $\mathrm{CdF}_{2}$ was measured by E. S. R. techniques. This dependence has been interpreted using the Simanek-Orbach theory.
\end{abstract}

Lors de leur étude par R. P. E. de l'ion $\mathrm{Mn}^{2+}$ dans $\mathrm{MgO}$, Walsh et al. [1] ont constaté une augmentation de la constante de couplage hyperfin $A$ quand la température décroissait. Ceci paraissait être en contradiction avec les résultats des études à pression variable qui donnaient une diminution de $A$ quand la pression augmentait.

Pour expliquer théoriquement cette variation de $A$ en fonction de la température, dans le cas des ions dans un état $\mathrm{S}$, les divers auteurs qui s'y sont intéressés [2], [3], [4] ont pris comme point de départ le couplage des électrons paramagnétiques avec les phonons du réseau. Ils ont, cependant, fait des hypothèses différentes en ce qui concerne les spectres de phonons.

Les résultats de ce travail concernent la variation $A(T)$ pour l'ion $\mathrm{Mn}^{2+}\left({ }^{6} \mathrm{~S}_{5 / 2}\right)$ dans $\mathrm{CdF}_{2}$. Ils ont été obtenus avec deux monocristaux d'origine différente. La valeur de la constante $A$ a été tirée de l'analyse des spectres de résonance basée sur la théorie de Low [5].

L'interprétation théorique de cette variation a été faite à l'aide de la méthode de Šimanek et Orbach [2]. Elle utilise un mélange de configurations excitées $3 \mathrm{~d}^{4} n$ s (avec $n \geqslant 4$ ) à la configuration fondamentale $3 \mathrm{~d}^{5}$ au moyen de l'interaction orbite-réseau de Van Vleck [6]. La variation du champ statique hyperfin $B_{\mathrm{c}}$ résultant de la polarisation du cœur de l'ion est définie comme étant l'élément de matrice :

$$
<{ }^{6} S^{\prime}\left|\frac{2}{3} \mu_{0} g_{e} \beta_{e} \sum_{i=1}^{5} S_{z i} \delta\left(\mathbf{r}_{i}\right)\right|{ }^{6} S^{\prime}>,
$$

que l'on pose égal à $\left\langle B_{\text {hyp }}\right\rangle$. La sommation se fait sur les cinq électrons $d$ de l'ion $\mathrm{Mn}^{2+}$.

L'état $\mid{ }^{6} S^{\prime}, M_{\mathrm{s}}=5 / 2>$ est un déterminant contenant cinq fonctions

$$
\begin{gathered}
\left|\psi_{3 \mathrm{~d},-2}^{\prime}\right\rangle,\left|\psi_{3 \mathrm{~d},-1}^{\prime}\right\rangle,\left|\psi_{3 \mathrm{~d}, 0}^{\prime}\right\rangle, \\
\left|\psi_{3 \mathrm{~d},+1}^{\prime}\right\rangle,\left|\psi_{3 \mathrm{~d},+2}^{\prime}\right\rangle
\end{gathered}
$$

REVUE DE PHYSIQUE APPLIQUÉE. - T. 6, No 2, JUIN 1971 perturbées au premier ordre par le couplage orbiteréseau $\mathrm{V}_{\mathrm{OR}}$, à savoir :

$$
\begin{aligned}
& \left|\psi_{3 \mathrm{~d}, \alpha}^{\prime}\right\rangle= \\
& =\left|\psi_{3 \mathrm{~d}, \alpha}\right\rangle+\sum_{n} \frac{\left\langle\psi_{n \mathrm{~s}}\left|\mathrm{~V}_{\mathrm{OR}}\right| \psi_{3 \mathrm{~d}, \alpha}\right\rangle}{E_{3 \mathrm{~d}, \alpha}-E_{n \mathrm{~s}}}\left|\psi_{n \mathrm{~s}}\right\rangle .
\end{aligned}
$$

$A$ étant proportionnel à $B_{\mathrm{c}}$ il est possible d'exprimer sa variation en fonction de la température par :

$$
A(T)=A(0)\left\{1-\frac{<B_{\text {hyp }}>}{B_{\mathrm{c}}}\right\} .
$$

En considérant les seuls phonons acoustiques de grande longueur d'onde $(\mathbf{k} . R \ll 1)$, le processus de Van Vleck pour les moyennes [7] et l'approximation du réseau d'ions ponctuels, la relation précédente se met sous la forme :

$$
A(T)=A(0)\left\{1-C . T^{4} \int_{0}^{\theta_{D / T}} \frac{x^{3} \mathrm{~d} x}{\mathrm{e}^{x}-1}\right\},
$$

où $\theta_{\mathrm{D}}$ est la température de Debye et $C$ une constante dépendant entre autres des vitesses longitudinale et transverse du son dans le matériau.

Cette loi est bien en accord avec l'expérience car si on ajuste $C$ pour une température donnée $\left(500^{\circ} \mathrm{K}\right.$ par exemple), la courbe de variation théorique passe par tous les points expérimentaux comme il est possible de le constater sur la figure $1\left(^{*}\right)$.

(*) Dans une publication parue juste avant ce Colloque, Huang et al. J. Phys. Chem. Sol., 1970, 31, 871, trouvent une variation $A(T)$ légèrement plus prononcée que la nôtre. $\mathrm{Ne}$ connaissant pas encore les constantes élastiques et la température de Debye de $\mathrm{CdF}_{2}$ ils ont évalué cette dernière à $350^{\circ} \mathrm{K}$ à partir de leur courbe expérimentale. 
Nous trouvons alors :

$$
A(0)=100,6 \mathrm{G}
$$

et $C_{\text {exp }}=2,1 \times 10^{-13}\left({ }^{\circ} \mathrm{K}\right)^{-4}$ ce qui est supérieur d'un ordre de grandeur à la valeur théorique

$$
C_{\text {théo }}=2,3 \times 10^{-14}\left({ }^{\circ} \mathrm{K}\right)^{-4}
$$

obtenue en utilisant les valeurs :

$$
\begin{aligned}
v_{1} & =5,46 \times 10^{3} \mathrm{~m} / \mathrm{s} \\
v_{\mathrm{t}} & =1,89 \times 10^{3} \mathrm{~m} / \mathrm{s} \\
\text { et } \quad \theta_{\mathrm{D}} & =328 \mathrm{o},
\end{aligned}
$$

déduites des résultats d'Alterovitz et Gerlich [8].

Un tel écart, commun aux différentes théories proposées [2], [3], [4] provient essentiellement de l'utilisation de l'approximation du réseau d'ions ponctuels.

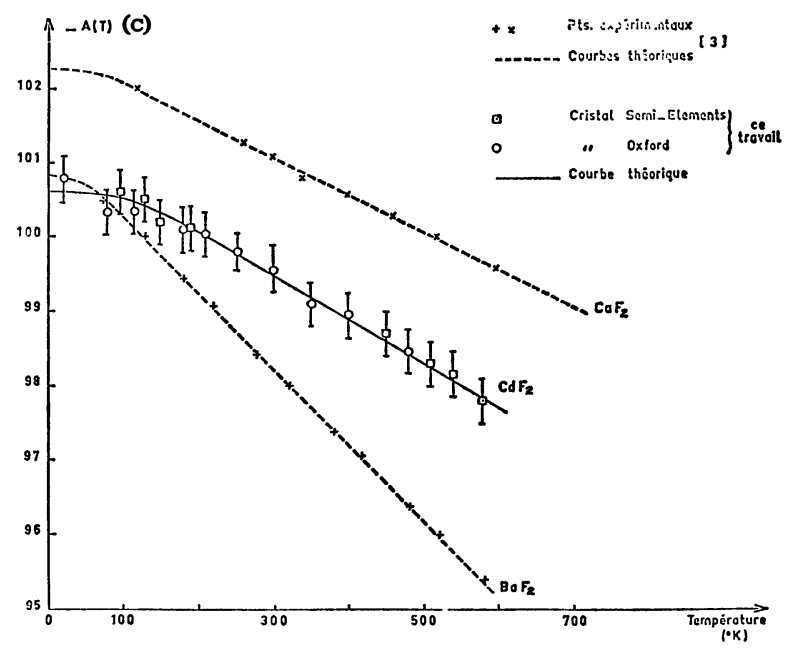

Fig. 1. - Variation de $A$ en fonction de la température pour $\mathrm{Mn}^{2+}$ dans $\mathrm{CaF}_{2}, \mathrm{BaF}_{2}$ et $\mathrm{CdF}_{2}$. La courbe en trait plein a été obtenue théoriquement à l'aide de l'équation (4) avec $C=2,1 \times 10^{-13}\left({ }^{\circ} \mathrm{K}\right)^{-4}$ et $\theta_{\mathrm{D}}=328^{\circ} \mathrm{K}$.

\section{Bibliographie}

[1] Walsh (W. M.), Jeener (J.) et Bloembergen (N.), Phys. Rev., 1961, 139A, 1338.

[2] Simanek (E.) et Orbach (R.), Phys. Rev., 1966, 145, 191. [3] Huang (C. Y.), Reichert (J. F.) et Gigante (J.), Phys. Letters, 1968, 26A, 219.

[4] Menne (T. J.), Phys. Rev., 1969, 180, 350.
[5] Low (W.), Phys. Rev., 1957, 105, 793.

[6] Van Vleck (J. H.), J. Chem. Phys., 1939, 7, 32.

[7] Van Vleck (J. H.), Phys. Rev., 1940, 57, 425.

[8] Alterovitz (S.) et Gerlich (D.), Phys. Rev., 1970, 1B, 4136. 